\title{
Research on Digital City Management Business System
}

Tao Guo

\author{
Longdong University, College of Information Engineering Gansu Qingyang 745000 \\ yzz_hyyf@163.com
}

Keywords: SOA; Digital city management; Business system

\begin{abstract}
With the continuous acceleration of the current urban development process, the digital city management system occupies a particularly important composition in the urban development. However, at this stage, it is more likely that there will be isolated islands of information among the application subsystems of all digital cities problem. This research aims at analyzing the digital management business system based on SOA, studying the multi-tier architecture of web service and SOA, discussing the integration of digital city business information, and can effectively realize the SOA-based digital management business system information integration based on web service system.
\end{abstract}

\section{Introduction}

In the current stage of social development, digital cities have been the building of relevant units in our country's society to gradually promote a new mode of management and application. By building a digital city, information integration can be better implemented for different application systems. Now Phase of the city's digital management business systems, usually using point-to-point information integration, such a complex approach is more likely to cause information silos. Service-oriented architecture application-integrated development program, you can effectively solve this problem, the different services can be shared between the portfolio. Such standardized service solutions can better cooperate with the original system to achieve efficient integration of information resources. In the current emergence of web services, SOA and the web is to create more for its development prospects.

\section{SOA Overview}

SOA is a service-oriented architecture that can be used by combining loosely coupled, coarse-grained, distributed implementations through a network based on specific needs. The service layer can be called directly in the application to achieve human-based dependencies in the control system and software. SOA is a component model that integrates and connects different services via functional definitions between different services. Interface is defined by means of neutrality, through the construction of service hardware platform, operating system and programming language. SOA-based system needs to be based on services, to achieve mutual synergies, independence, modularity, clarity, in order to achieve can be found, reusable and many other features.

\section{SOA Technology}

Web Seivices is a particularly suitable application of SDA technology, through a distributed application architecture built on top of XML, UDDI, SOAP, and WSDL. Based on its functional nature, services are made available by Web Seivices based on their business logic, which can be published and referenced on the Internet platform. Three roles are described in Web Services, including service providers, service requesters and service agents. Service providers implement web function owners, provide other services with their own functions, and service requesters implement The user of the web service function sends the information through the SOAP message to obtain the corresponding function service. Service agents are mainly the middle of the web function to achieve, but also act as managers of the more color (as shown in Figure 1). 


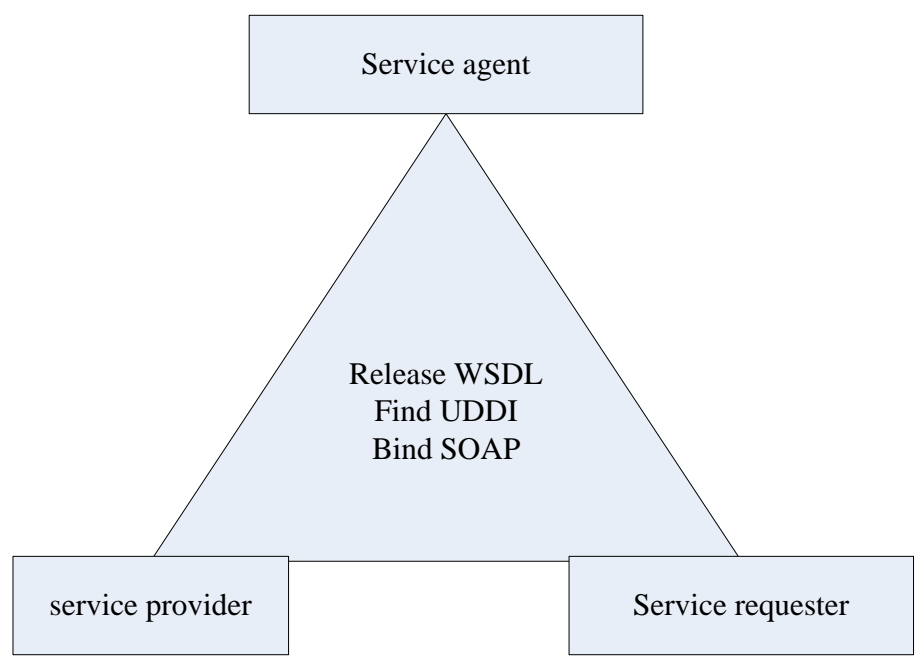

Figure 1. Web service architecture diagram

\section{SOA Analysis and Design}

\section{SOA Analysis Goals and the Corresponding Process}

Through analysis of multidimensional services, the main purpose is to determine how to make use of service-oriented and corresponding ways to accomplish the service requirements of business automation. The purpose of the service-oriented phasing is to enable subsequent functional projects to be identified. In the analysis of services to achieve the process, usually need to analyze specific service goals. Such phases usually encounter different problems, such as which services need to be completed and which logical encapsulation needs to be completed in completing the service. The main scope of the original problem needs to be directly determined during the analysis. Then the specific analysis flow chart (shown in Figure 2).

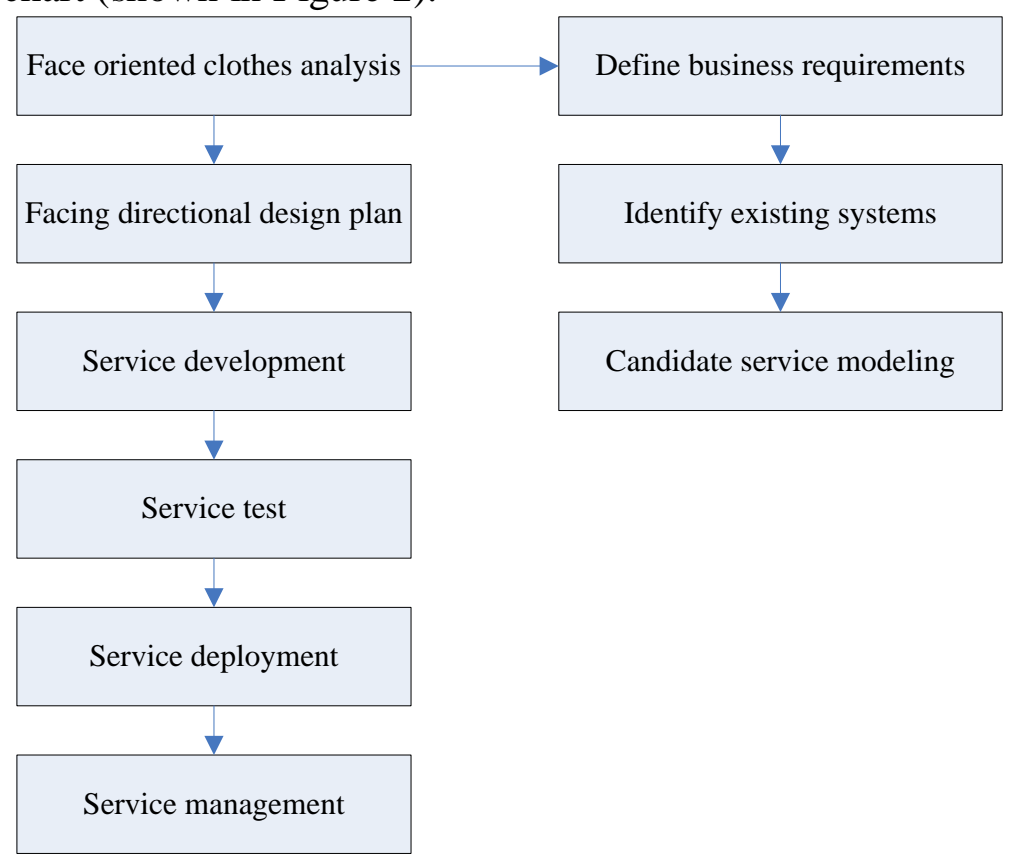

Figure 2. High-level service-oriented analysis of the flow diagram

\section{SOA Design}

SOA is a kind of organizational design method based on specific indicators. Based on the traditional business and technology, SOA can build a connection-based and service-specific service function and effectively achieve the software technology architecture of the entire business system. Through the use of Web Seivices to achieve the effective communication of different levels of 
functional information, the application layer to get rid of the original technical features of the binding, so as to better the system functions in the overall service level, so that the entire business system can be more rich, flexible, Focus, at the same time more able to have a stronger business purpose. The specific design of SOA is divided into several steps to complete (as shown in Figure $3)$.

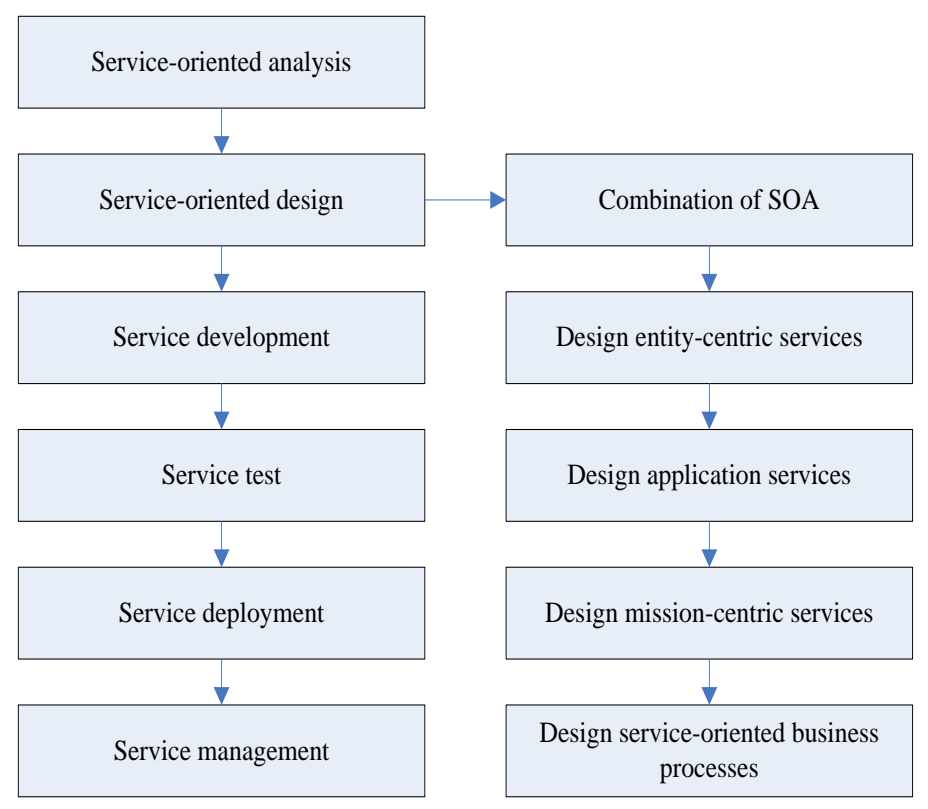

Figure 3. High-level service-oriented design process diagram

\section{NET Service Framework SOA}

NET is Microsoft's multi-language feature development tool with powerful system and completeness. The tool possesses strong operation convenience, and can develop many desktop application programs, ASP.NET programs, mobile programs As well as the degree of web services and other functions, by using NET tools to develop service framework, you can more easily complete the development of SOA features, debugging, testing and functional deployment. Server-side infrastructure, are based on ASP.NET achieved more convenient to achieve the functional integration of information, including restart and cache functions. In addition to or absorb the Session Application function simplification, as well as ASP.NET framework services object function. This built-in object can always maintain the web service object, and multiple users and applications to maintain state storage, interaction, and thus complete NET services within the framework of transmission and communication.

\section{Based on SOA Digital City Management Business System Functional Structure}

At this stage in the realization of digital city management business system process, the market SOA solutions include three: one is based on IBM architecture, the other is based on NET architecture, the third is based on SAP architecture, the city Digital management business system involves more departments, and business information is also particularly complex, so by using the second as the main support structure.

At the access layer, diversified access methods for city business management structures, including websites, telephones, and city administrations, are implemented at the access layer. Information on urban management, such as the public, media organizations and government departments, is reflected in various aspects of urban management. The second is the business process layer. This layer realizes the information analysis of the business management system of the city, and transforms the complex information and numbers into a structured business process, thus realizing the modeling, identification, development and deployment among the agencies. Furthermore, the service layer includes a web service platform between multiple business process layers to achieve coarse-grained business functions, ensure the details achieved between the various 
functions, ensure the specific business process dynamics in the business process layer, and ensure that the service layer The overall data security sharing. Then it is the application layer, which can implement various aspects of system management, including the relevant information obtained by multiple departments to ensure the information can be operated anytime and anywhere, and provide support for city-related decision-making.

\section{Conclusion}

SOA-based digital city management business systems, SOA can effectively solve the problem of information exchange between different systems, this study through SOA-specific urban management of digital business system design and functional structure analysis of the development and found that SOA can be effective To meet the business management of the city, have some reference to the research on SOA in the future.

Project Fund:

2018 Gansu natural science fund: Popularization and Application of 3D Digital Community Refined Management Service System

2018 science and technology planning project of Xifeng District, Qingyang City: Innovation and Practice of the Management Mode of Smart Community on East Avenue

Science and technology research project of Longdong University: Promotion and Application of 4D Community Digital Map and Data Management System in Xifeng District

\section{References}

[1] Botto-Tobar M, Ramirez-Anormaliza R, Cevallos-Torres L J, et al. Migrating SOA Applications to Cloud: A Systematic Mapping Study[C]// International Conference on Technologies and Innovation. Springer, Cham, 2017:3-16.

[2] Jamshidi P, Ahmad A, Pahl C. Cloud Migration Research: A Systematic Review[J]. IEEE Transactions on Cloud Computing, 2014, 1(2):142-157.

[3] Botto-Tobar M, Ramirez-Anormaliza R, Cevallos-Torres L J, et al. Migrating SOA Applications to Cloud: A Systematic Mapping Study[M]// Technologies and Innovation. 2017.

[4] Haddad L, Decimoni T, Turri A, et al. PGI32-Economic Evaluation Studies In Gastroenterology In Brazil: A Systematic Review[J]. Value in Health the Journal of the International Society for Pharmacoeconomics \& Outcomes Research, 2014, 17(7):A368.

[5] Foundation SOA high school numbering school system systematic research assignment [D] Yamada University, 2015.

[6] Zhongwei, Lee Yong Feng, Yu Bin. Basis SOA Numerical Standards Assembly Hiradaya Design Assignment Effectiveness - State State Standardization Standards Control and Trust Bureau Activity Examples [J] Castle City Regulations, 2011, 35 (7): 89-92. 